\title{
An Introduction to Numerical Methods for the Solutions of Partial Differential Equations
}

\author{
Manoj Kumar, Garima Mishra \\ Department of Mathematics, Motilal Nehru National Institute of Technology, Allahabad, India \\ E-mail:manoj@mnnit.ac.in,garima.iitg08@gmail.com \\ Received August 8, 2011; revised September 27, 2011; accepted October 5, 2011
}

\begin{abstract}
Partial differential equations arise in formulations of problems involving functions of several variables such as the propagation of sound or heat, electrostatics, electrodynamics, fluid flow, and elasticity, etc. The present paper deals with a general introduction and classification of partial differential equations and the numerical methods available in the literature for the solution of partial differential equations.
\end{abstract}

Keywords: Partial Differential Equations, Eigenvalue, Finite Difference Method, Finite Volume Method, Finite Element Method

\section{Introduction}

An equation involving derivatives or differentials of one or more dependent variables with respect to one or more independent variables is called a differential equation. The study of differential equations is a wide field in pure and applied mathematics, physics, meteorology, and engineering, etc. All of these disciplines are concerned with the properties of differential equations of various types. Pure mathematics focuses on the existence and uniqueness of solutions, while applied mathematics emphasizes the rigorous justification of the methods for approximating solutions. Differential equations play an important role in modeling virtually every physical, technical, or biological process, from celestial motion, to bridge design, and interactions between neurons. Differential equations which are used to solve real-life problems may not necessarily be directly solvable, that is, do not have closed form solutions. Instead, solutions can be approximated using numerical methods. Mathematicians also study weak solutions (relying on weak derivatives), which are types of solutions that do not have to be differentiable everywhere. This extension is often necessary for solutions to exist, and it also results in more physically reasonable properties of solutions, such as possible presence of shocks for equations of hyperbolic type.

The theory of differential equations is quite developed and the methods used to study them vary significantly with the type of the equation.
A differential equation involving derivatives with respect to single independent variable is called an ordinary differential equation. In the simplest form, the dependent variable is a real or complex valued function, but more generally, it may be vector-valued or matrix-valued: this corresponds to considering a system of ordinary differential equations for a single variable. Ordinary differential equations are classified according to the order of the highest derivative of the dependent variable with respect to the independent variable appearing in the equation. The most important cases for applications are first-order and second-order differential equations. In the classical literature, the distinction is also made between differential equations explicitly solved with respect to the highest derivative and differential equations in an implicit form.

A differential equation involving partial derivatives with respect to two or more independent variables is called partial differential equation. The partial differential equations can also be classified on basis of highest order derivative.

Some topics in differential geometry as minimal surfaces and imbedding problems, which give rise to the Monge-Ampere equations, have stimulated the analysis of partial differential equations, especially nonlinear equations. Moreover, the theory of systems of first order partial differential equations has a significant interaction with Lie theory and with the work of E. Cartan.

The development of partial differential equations in the 18th and 19th century is given in Kline's book [1]. 
Until the 1870, the study of partial differential equation was mainly concerned with heuristic methods for finding solutions of boundary value problems as well as explicit solutions for particular problems (for an example, the solution of Dirichlet boundary value problem $\Delta u=0$, $u$ in $\Omega \subset \mathbb{R}^{2}$, introduced by Riemann).

Poincaré [2] gave the first complete proof of the existence and uniqueness of a solution of the Laplace equation for any continuous Dirichlet boundary condition in 1890. In a fundamental paper of, Poincare [3] established the existence of an infinite sequence of eigenvalues and corresponding eigen-functions for the Laplace operator under the Dirichlet boundary condition. Picard applied the method of successive approximation to obtain solutions of nonlinear problems which were mild perturbations of uniquely solvable linear problems. The construction of elementary solutions and Green's functions for general higher order linear elliptic operators was carried through to the analytic case by E. E. Levi [4]. Up to about 1920's solutions of partial differential equations were generally understood to be classical solutions, that is, $C^{k}$ for a differential operator of order $k$.

Keeping in view the requirement of the new researchers, the present paper describes the basic fundamentals of partial differential equations which has been collected from a large number of research articles published in reputed journals and literature available in the books with the intension to provide all important relevant material in a condense form related to partial differential equations and numerical methods for their solutions. Also, since analytical and computational solution of partial differential equations is the major concern from the early years, this paper gives a small step towards the development of computational analysis of partial differential equations, which have lot of utilization in the field of science and engineering.

\section{Classification of Partial Differential Equations}

Both ordinary and partial differential equations are broadly classified as linear and nonlinear. A linear partial differential equation is one in which all of the partial derivatives appears in linear form and none of the coefficients depends on the dependent variables. The coefficient may be function of the independent variables. A nonlinear partial differential equation can be described as a partial differential equation involving nonlinear terms.

\subsection{Types of Non-Linear Partial Differential Equations}

The non-linear partial differential equations describe many different physical systems, ranging from gravitation to fluid dynamics and have been used in mathematics to solve problems such as Poincare conjecture and Calabi conjecture.

A non-linear partial differential equation is known as semi-linear if it is linear in highest order derivatives and the coefficients of highest order derivatives depend only on independent variables.

$$
\sum_{|\beta|=k} a_{\beta} D^{\beta} u+a_{0}\left(D^{k-1} u, \cdots, D u, u, x\right)=0 .
$$

A nonlinear partial differential equation is known as quasi-linear if it is linear in highest order derivatives and the coefficients of highest order derivatives depend on independent variables as well on lesser order derivatives.

$$
\begin{aligned}
& \sum_{|\beta|=k} a_{\beta}\left(D^{k-1} u, \cdots, D u, u, x\right) D^{\beta} u \\
& \quad+a_{0}\left(D^{k-1} u, \cdots, D u, u, x\right)=0 .
\end{aligned}
$$

A nonlinear partial differential equation is known as fully non-linear if the coefficients depends on dependent variable or the derivatives appear in nonlinear form.

Example 2.1

$$
\begin{gathered}
f_{x x}+f_{y y}+f_{x}+f_{y}=f+x y \text { is linear equation } \\
a f_{x x}+b f_{y y}+f_{x}^{2}+f_{y}=c \text { is semilinear } \\
f_{x} f_{x x}+f_{y} f_{y y}+f_{x}^{2}+f=0 \text { is quasilinear } \\
f f_{x x}+f_{y y}+a f_{x}+b f_{y}=0 \text { is nonlinear }
\end{gathered}
$$

where $a, b$ are functions of $x, y$ and $c$ is function of $x, y$ and $f$.

But further classification into elliptic, hyperbolic, and parabolic equations, especially for second-order linear equations, is of utmost importance. For more study on linear and quasi linear elliptic equations, see $[5,6]$.

\subsection{Classification Based on Discriminant}

The general quasi linear second-order non-homogeneous partial differential equation in two independent variable is

$$
A f_{x x}+B f_{x y}+C f_{y y}+D f_{x}+E f_{y}+F f=G
$$

The classification of partial differential equations depends on the sign of discriminant $B^{2}-4 A C$ as fallow:

1) If $B^{2}-4 A C>0$, the partial differential equation is hyperbolic.

2) If $B^{2}-4 A C=0$, the partial differential equation is parabolic.

3) If $B^{2}-4 A C<0$, the partial differential equation is elliptic. 


\subsection{Eigenvalue Based Classification of Partial Differential Equations}

Since method for classification of partial differential equations fails if it is partial differential equation in three or more independent variable then we are not able to find discriminant.

Let us consider a general second order partial equation in $\mathrm{n}$ independent variables

$$
\begin{array}{cccccc}
a_{11} u_{x_{1} x_{1}} & +a_{12} u_{x_{1} x_{2}}+a_{13} u_{x_{1} x_{3}}+\ldots & +a_{1 n} u_{x_{1} x_{n}}+ \\
a_{21} u_{x_{2} x_{1}} & +a_{22} u_{x_{2} x_{2}}+a_{23} u_{x_{2} x_{3}}+\ldots & +a_{2 n} u_{x_{2} x_{n}}+ \\
\vdots & \vdots & \vdots & \ddots & \vdots \\
a_{n 1} u_{x_{n} x_{1}}+a_{n 2} u_{x_{n} x_{2}}+a_{n 3} u_{x_{n} x_{3}}+\ldots & +a_{n n} u_{x_{n} x_{n}}+ \\
b_{1} u_{x_{1}}+b_{2} u_{x_{2}}+b_{3} u_{x_{3}}+\ldots+b_{n} u_{x_{n}}+c u=0 .
\end{array}
$$

which also can be written in compact form as:

$$
\sum_{i=1}^{n} \sum_{j=1}^{n} a_{i j} \frac{\partial^{2} u}{\partial x_{i} x_{j}}+\sum_{i=1}^{n} b_{i} \frac{\partial u}{\partial x_{i}}+c u=0 .
$$

Then coefficient matrix of highest order derivatives is

$$
A=\left[a_{i j}\right] .
$$

Let $\lambda$ be an eigenvalue of $A$ corresponding to eigenvector $X$

$$
\begin{gathered}
A X=\lambda X \\
A X-\lambda X=0 \\
(A-\lambda I) X=0 \\
|A-\lambda I|=0 \quad \text { Since }\|\mathrm{X}\| \neq 0
\end{gathered}
$$

then

$$
\left|\begin{array}{cccc}
a_{11}-\lambda & a_{12} & \ldots & a_{1 n} \\
a_{21} & a_{22}-\lambda & \ldots & a_{2 n} \\
\vdots & \vdots & \ddots & \vdots \\
a_{n 1} & a_{n 2} & \ldots & a_{n n}-\lambda
\end{array}\right|=0 .
$$

The characterisic equation will have $n$ roots as $n$ eigenvalues.

Characterization of differential equation is based on following:

- If any eigenvalue is zero, then partial differential equation is parabolic.

- If all eigenvalues are non-zero and one eigenvalue has opposite sign, then partial differential equation is hyperbolic.

- If all eigenvalues are nonzero having same sign, then partial differential equation is elliptic.

Example 2.2 Consider the following differential equation of flow:

$$
\left(1-M_{\infty}^{2}\right) \frac{\partial^{2} \phi}{\partial x^{2}}+\frac{\partial^{2} \phi}{\partial y^{2}}=0
$$

where $M_{\infty}$ is Mac number.

1) First we characterize by discriminant

$$
\begin{aligned}
& B=0, A=1-M_{\infty}^{2}, C=1, \\
& \text { then } B^{2}-4 A C=-4\left(1-M_{\infty}^{2}\right)=4\left(M_{\infty}^{2}-1\right) .
\end{aligned}
$$

- If flow is subsonic i.e. $M_{\infty}<1$, then

$$
B^{2}-4 A C<0 \text {. }
$$

The equation of flow is elliptic.

- If flow is sonic i.e. $M_{\infty}=1$, then

$$
B^{2}-4 A C=0 \text {. }
$$

The equation of flow is parabolic.

- If flow is subsonic i.e. $M_{\infty}>1$, then

$$
B^{2}-4 A C>0 \text {. }
$$

The equation of flow is hyperbolic.

2) Now by eigenvalues

$$
\begin{gathered}
A=\left[\begin{array}{cr}
1-M_{\infty}^{2} & 0 \\
0 & 1
\end{array}\right] \\
|A-\lambda I|=\left|\begin{array}{cc}
\left(1-M_{\infty}^{2}\right)-\lambda & 0 \\
0 & 1-\lambda
\end{array}\right| \Rightarrow \lambda=1,\left(1-M_{\infty}^{2}\right) .
\end{gathered}
$$

- If $M_{\infty}<1$, then all eigenvalues are nonzero and of same sign thus equation of flow is elliptic.

- If $M_{\infty}=1$, then all eigenvalues are zero thus equation of flow is parabolic.

- If $M_{\infty}>1$, then all eigenvalues are nonzero and of one eigenvalue of opposite sign thus equation of flow is hyperbolic.

\subsection{Significance of Classification}

The classification of a partial differential equations is intimately related to the characteristics of the partial differential equations. The characteristics are $(n-1)$ dimensional hyper-surfaces in $n$-dimensional hyperspace that have some very special features. In two-dimensional space, which is the case considered generally, characteristics are paths in the solution domain along which information propagates. In other words we can say information propagates throughout the solution domain along characteristics.

\subsection{Classification by Physical Problems}

Physical problems falls into one of the following general classification:

1) Equilibrium Problem;

2) Propagation Problem;

3) Eigenvalue Problem.

Equilibrium Problem: Equilibrium problems are steady state problems in closed domain $D(x, y)$ in which the solution $f(x, y)$ is governed by an elliptic 
partial differential equations subject to boundary conditions specified at each point on the boundary $B$ of the domain.

Such type of problems have no real characteristic and thus the solution at every point in the solution domain is influenced by the solution at all other points and the solution at each points influence the solution at all other points.

Equilibrium problems are solved by method of relaxation numerically.

Propagation Problem: Propagation problems are initial value problems in open domains. Here by open domain means that open with respect to one of the independent variables.

\section{Example 2.3}

$$
f_{t}=\alpha f_{x x} \text { with initial condition } f\left(x, t_{0}\right)=f(x) .
$$

Eigenvalue Problem: Eigenvalue problem are special problems in which the solution exits only for special values (i.e. eigenvalues) of a parameter of the problem. The eigenvalues are to be determined in addition to the corresponding configuration of the system.

\subsection{Types of Conditions}

Initial Value Problem: An initial value problem is one in which the dependent variable and possibly its derivatives are specified initially (i.e. at time $t=0$ ) or at the same value of independent variable in the equation. Initial value problems are generally time-dependent problems.

$$
\text { Example 2.4 } \frac{\partial u}{\partial t}=\alpha \frac{\partial^{2} u}{\partial^{2} x}, u\left(x, t_{0}\right)=f(x) \text {. }
$$

Boundary Value Problems: A boundary value problem is one in which the dependent variable and possibly its derivatives are specified at the extreme of the independent variable. For steady state equilibrium problems, the auxiliary conditions consists of boundary conditions on the entire boundary of the closed solution domain. There are three types of boundary condition.

Example 2.5 Let $\Omega$ be a bounded domain in $\mathbb{R}^{n}$ with a smooth boundary $\partial \Omega$, and let $f: \bar{\Omega} \times \mathbb{R} \rightarrow \mathbb{R}$ be a locally Hölder continuous function. The BVP

$$
\begin{gathered}
-\Delta u=f(x, u), \text { in } \Omega \\
u=0, \text { on } \partial \Omega
\end{gathered}
$$

is called nonlinear elliptic boundary value problem. This type of BVP arises in several domains, for example in physical problems involving the steady-state temperature distribution see [7-9].

1) Dirichlet boundary condition: The value of the function $f$ is specified on the boundary. The dependent variable of the partial differential equation are pre- scribed in domain at different points. For example if an iron rod had one end held at absolute zero then the value of the problem would be known at that point in space. A Dirichlet boundary condition imposed on an ordinary or a partial differential equation specifies the values of a solution is to take on the boundary of the domain. The question of finding solutions to such equations is known as the Dirichlet problem as in (10).

2) Neumann boundary condition: The value of derivative normal to the boundary is specified $\left(\frac{\partial f}{\partial n}\right.$ is specified on the boundary). For example if one iron rod had heater at one end then energy would be added at a constant rate but the actual temperature would not be known. A Neumann boundary condition imposed on an ordinary or a partial differential equation specifies the derivative values of a solution is to take on the boundary of the domain. For example

$$
\begin{gathered}
-\Delta u=f(x, u), \text { in } \Omega \\
\frac{\partial u}{\partial x}=0, \text { on } \partial \Omega .
\end{gathered}
$$

3) Mixed boundary condition: The linear combination of Dirichlet and Neumann boundary conditions:

$$
a f+b \frac{\partial f}{\partial n}
$$

is specified on the boundary.

Mixed boundary conditions are also known as Cauchy boundary condition. A Cauchy boundary condition imposed on an ordinary or a partial differential equation specifies both the values a solution of a differential equation is to take on the boundary of the domain and the normal derivative at the boundary.

$$
\begin{gathered}
-\Delta u=f(x, u), \text { in } \Omega \\
c_{1} \frac{\partial u}{\partial x}+c_{2} u=0, \text { on } \partial \Omega .
\end{gathered}
$$

\section{Various Methods for Solving Partial Differential Equation}

In literature various method exits for solution of partial differential equations. Here we will discuss some of them briefly as following:

1) Finite Difference Method: The finite difference method is a numerical procedure which solves a partial differential equation by discretizing the continuous physical domain into a discrete finite difference grid, approximating the individual exact partial derivatives in the partial differential equations by algebraic finite difference approximations (i.e. FDA), substituting the FDA's into the partial differential equations to obtain an alge- 
braic finite difference equation(FDE), and solving the resulting algebraic finite difference equations for the dependent variable.

For detailed study on finite difference method, we encourage to readers to consult the references [10-19].

2) Finite Volume Method: The finite volume method is a method for representing and evaluating partial differential equations in the form of algebraic equations. Similar to the finite difference method, values are calculated at discrete places on a meshed geometry. "Finite volume" refers to the small volume surrounding each node point on a mesh. In the finite volume method, volume integrals in a partial differential equation that contain a divergence term are converted to surface integrals, using the divergence theorem. These terms are then evaluated as fluxes at the surfaces of each finite volume. Because the flux entering a given volume is identical to that leaving the adjacent volume, these methods are conservative. The method is used in many computational fluid dynamics packages

One advantage of the finite volume method over finite difference methods is that it does not require a structured mesh (although a structured mesh can also be used). Furthermore, the finite volume method is preferable to other methods as a result of the fact that boundary conditions can be applied non-invasively. This is true because the values of the conserved variables are located within the volume element, and not at nodes or surfaces. Finite volume methods are especially powerful on coarse nonuniform grids and in calculations where the mesh moves to track interfaces or shocks.

For more details on finite volume method, we refer to [20-22].

3) Finite Element Method: The finite element method, where functions are represented in terms of basis functions and the partial differential equations is solved in its integral (weak) form. In the finite element method (FEM) the domain $\Omega$ is partitioned in a finite set of elements $\left\{\Omega_{i}\right\}$, so that $\left\{\Omega_{i} \cap \Omega_{j}\right\}=\phi$ for $i \neq j$, and $\cup \bar{\Omega}_{i}=\bar{\Omega}$. Usually one takes for $\Omega_{i}$ triangles or quadrangles. Then the function is approximated by $u_{h}=\sum a_{i} \phi_{i}(x)$, where $\phi_{i}$ are functions that are polynomials on each $\phi_{i}$ (i.e. piecewise polynomials). Usually the functions $\phi_{i}$ are continuous polynomials of a low degree. Further they are constructed so that their support extends only over a small number of elements.

Now we will give detail discussion of Finite element method.

\section{Finite Element Method}

The key idea: The finite element is a numerical method like finite difference method but it is more general and powerful in its application to real-world problems that involve complicated physical geometry and boundary conditions.

In FEM, a given domain is viewed as a collection of sub-domains, and over each sub-domain the governing equation is approximated by any of the traditional variational methods.

The main reason behind taking approximate solution on a collection of sub-domains is the fact that it is easier to represent a complicated function as a collection of simple polynomials.

The method is characterized by three features:

1) The domain of the problem is represented by a collection of simple sub-domains called finite elements. The collection of finite elements is called the finite element mesh.

2) Over each finite element, the physical process is approximated by functions of the desired type and algebraic equations relating physical quantities at selective points, called nodes of the element are developed.

3) The element equation are assembled using continuity and/or "balance" of physical quantities.

In FEM, we seek an approximation $u_{n}$ of $u$ in the form

$$
u \approx u_{n}=\sum_{j=1}^{n} u_{j} \Psi_{j}+\sum_{j=1}^{n} c_{j} \phi_{j}
$$

where $u_{j}$ are the values of $u_{n}$ at the element nodes $\Psi_{j}$ are the interpolation function, $c_{j}$ are coefficients that are not associated with nodes, and $\pi_{j}$ are the associated approximation functions. Direct substitution of the such approximation into the governing differential equation does not always result, for an arbitrary choice of the data of the problem, in a necessary and sufficient no. of equations for the under-determined coefficients $u_{j}$ and $c_{j}$ Therefore a procedure whereby a necessary and sufficient number of equations can be obtained is needed.

There is only one method of finite element model of the same problem. There can be more than one finite element model of the same problem. The type of model depends on the differential equations, method used to derive the algebraic equations for the undetermined coefficients over an element, and nature of the approximations function used.

\subsection{Variational Principles and Methods}

The idea of using a variational formulation of a boundary value problem for its numerical solution goes back to Lord Rayleigh $(1894,1896)$ and Ritz (1908), see, e.g., Kantorovich and Krylov [13]. In Ritz's approach the approximation solution was sought as a finite linear com- 
bination of functions such as, for instance, polynomial or trigonometric polynomials. The use in this context of continuous piecewise linear approximating function based on triangulation adapted to the geometry of the domain proposed by Courant (1943) in the paper based on an address delivered to the American Mathematical Society in 1941. Even though this idea had appeared earlier, also in work by Courant himself (see Babuska [23]), this is often thought as the starting point of the finite element method, but the further development and analysis of the method would occur much later.

Classical sense of the variational principle is to find the extremum or the variables of the problem. The functional includes all the intrinsic features of the problem such as the governing equations, boundary conditions, and constraints condition, if any.

In solid and structural mechanics problems, the functional represents the total energy of the system and in other problems it is simply an integral representation of the governing equations.

First many problems of mechanics are posed in terms of finding the extremum and thus by their nature, can be formulated in terms of variational statement. Second, there are problems that can be formulated by other means, but these can also be formulated by means of variational principles. Third, variational formulations form a powerful basis for obtaining approximate solutions to practical problems, many of which are intractable otherwise. The principle of minimum total potential energy, for example, can be regarded as a substitute to the equations of equilibrium of an elastic body as well as a basis for the development of displacement finite element models that can be used to determine approximate displacement and stress fields in the body.

\subsection{Variational Formulations}

The classical sense of the phrase "Variational Formulation" refers to the construction of a functional or variational principle that is equivalent to the governing equations of the problem. The modern use of the phrase refers to the formulation in which the governing equations are translated into equivalent weighted integral statements that are not necessarily equivalent to a variational principle.

The importance of variational formulation of physical laws, in the modern or general sense of the phrase, goes for beyond its use as simply an alternate to other formulation. In fact, variational forms of the laws of continuum physics may be only natural and rigorously correct way to think of them. While all sufficiently smooth fields leads to meaningful variational forms the converse is not true. There exits physical phenomena which can be ade- quately modeled mathematically only in a variational setting they are nonsensical when viewed locally.

The starting point for the discussion of the finite element method is differential equations governing the physical phenomena under study. As such, we shall first discuss why integral statement of differential equations are needed.

\subsubsection{Need for Weighted-Integral Statements}

The weighted-integral statement are required in order to generate the necessary and sufficient number of algebraic equations to solve for the parameters $c_{j}$ in Equation (13) of approximate solution.

$$
u(x) \approx u_{N}=\sum_{j=1}^{N} c_{j} \phi_{j} .
$$

The use of integral statement is equivalent to the governing differential equation is necessitated by the fact that substitution of Equation (13) into the governing differential does not always results in the required number of linearly independent algebraic equations for the unknown coefficients $c_{j}$. One way to insure that there are exactly the same number $n$ of equations as there are unknowns is to require weighted integrals of the error in the equation to be zero. We can require the approximate solution $u$ to satisfy the given differential equation in the weighted integral sense,

$$
\int_{\Omega} w R \mathrm{~d} x=0
$$

where $R$ is called residual.

\subsubsection{Linear and Bilinear Functional}

Consider the integral expression

$$
I(u)=\int_{a}^{b} F\left(x, u, u^{\prime}\right) \mathrm{d} x, u=u(x), u^{\prime}=\frac{\mathrm{d} u}{\mathrm{~d} x} .
$$

For a given real function $u=u(x), I(u)$ is a real number. Therefore, $I$ can be viewed as an operator that transforms functions $u(x)$ into real numbers, and such operators called "functionals".

\section{Example 4.1}

$$
\begin{gathered}
I(u)=\int_{a}^{b}\left(P(x) \frac{\mathrm{d} u}{\mathrm{~d} x}+q(x) u^{2}\right)+P u(a) . \\
I(u, v)=\int_{\Omega}\left(P(x, y) \frac{\mathrm{d} u}{\mathrm{~d} x} \frac{\mathrm{d} v}{\mathrm{~d} x}+q(x, y) v\right) \mathrm{d} x \mathrm{~d} y+\int_{\Gamma} Q u \mathrm{~d} s .
\end{gathered}
$$

A functional $l(u)$ is said to be linear in $u$ if and only if it satisfies the relation

$$
l(\alpha u+\beta v)=\alpha l(u)+\beta l(v) .
$$

for any real numbers $\alpha$ and $\beta$ and dependent variable $u$ and $v$. 


\section{Example 4.2}

1) $I(u)=\int_{a}^{b} f(x) u \mathrm{~d} x+q(x) u(b)$.

2) $I(u, v)=\int_{\Omega}(f(x, y) u+q(x, y) v) \mathrm{d} x \mathrm{~d} y$.

A function $B(u, v)$ is said to be bilinear if it is linear in each of its arguments $u$ and $v$ :

$$
B\left(\alpha u_{1}+\beta u_{2}, v\right)=\alpha B\left(u_{1}, v\right)+\beta B\left(u_{2}, v\right)
$$

linear in first argument.

$$
B\left(u, \alpha v_{1}+\beta v_{2}\right)=\alpha B\left(u, v_{1}\right)+\beta B\left(u, v_{2}\right)
$$

linear in second argument.

A bilinear form $B(u, v)$ is said to be symmetric in its arguments $u$ and $v$ if

$$
B(u, v)=B(v, u), \text { for all } u, v .
$$

\subsubsection{Variational Operator and First Variation}

Consider the function $F\left(x, u, u^{\prime}\right)$. For any fixed value of the independent variable $x, F$ depends on $u$ and $u^{\prime}$. The change $\varepsilon v$ in $u$ where $\varepsilon$ is a constant quantity and $v$ is a function of $x$ is called the variation of $u$ and is defined by $\delta u$

$$
\delta u=\varepsilon v .
$$

$\delta$ is called variational operator.

The variation $\delta u$ of a function u represents an admissible change in the function $u(x)$ at a fixed value of the independent variable $x$. If $u$ is specified value cannot be varied. Thus, the variation of a function $u$ is zero there because the specified on the boundary, the variation of $u$ is zero there because the specified value cannot be varied. Thus, the variation of a function $u$ should satisfy the homogeneous form of the boundary conditions for $u$

$$
\Delta F=F\left(x, u+\varepsilon v, u^{\prime}+\varepsilon v\right) .
$$

Fundamental lemma of variational calculus: The fundamental lemma of variations can be stated as follows for any integrable function $G(x)$, if the statement

$$
\int_{a}^{b} G(x) \eta(x) \mathrm{d} x=0,
$$

holds for any arbitrary function $\eta(x), \forall x \in(a, b)$, then it follows that $G(x)=0$ in $(a, b)$.

General statement of fundamental lemma is as following:

If $\eta(x)$ is arbitrary in $a<x<b$ and $\eta(a)$ is arbitrary then the statement

$$
\begin{aligned}
& \int_{a}^{b} G \eta(x) \mathrm{d} x+B(a) \eta(a)=0 \\
& \Rightarrow G=0 \text { in } a<x<b \text { and } B(a)=0
\end{aligned}
$$

because $\eta(x)$ is independent of $\eta(a)$.

\subsubsection{Natural and Essential Boundary Conditions}

Essential boundary conditions which require $v$ and possible its derivatives to vanish at the boundary. Thus, we have

$$
\text { Specify } v=0 \text {, or } u=\hat{u} \text { on the boundary. }
$$

These conditions are also known as Dirichlet or geometric boundary conditions.

Natural boundary condition, which require specification of the coefficient of $v$ (and possibly its derivatives). Thus we have

$$
\text { Specify } \frac{\partial F}{\partial u^{\prime}}=Q, \text { on the boundary. }
$$

Natural boundary conditions are also known as Neumann boundary conditions.

Example 4.3 Finding a function $u=u(x)$ such that $u(a)=u_{a}, u(b)=u_{b}$ and

$$
\begin{array}{r}
I(u)=\int_{a}^{b} F\left(x, u(x), u^{\prime}(x)\right) \mathrm{d} x \text { is a minimum } \\
F\left(x, u, u^{\prime}\right)=\frac{\partial F}{\partial u}-\frac{\mathrm{d}}{\mathrm{d} x}\left(\frac{\partial F}{\partial u^{\prime}}\right)=0 \text { in } a<x<b .
\end{array}
$$

The necessary condition for $I$ to attain a minimum yields

$\int_{a}^{b} v\left[\frac{\partial F}{\partial u}-\frac{\mathrm{d}}{\mathrm{d} x}\left(\frac{\partial F}{\partial u^{\prime}}\right)\right] \mathrm{d} x+\left(\frac{\partial F}{\partial u^{\prime}} v\right)_{a}^{b}-Q_{a} v(a)-Q_{b} v(b)=0$.

Now suppose that $\frac{\partial F}{\partial u^{\prime}}$ and $v$ are selected such that

$$
\left(-\frac{\partial F}{\partial u^{\prime}}-Q_{a}\right) v=0 \text { for } x=a,\left(\frac{\partial F}{\partial u^{\prime}}-Q_{b}\right) v=0 \text { for } x=b .
$$

Then using the fundamental lemma of the calculus of variations, we obtain the same Euler equation.

Equations in (29) are satisfied identically for any of the following combination:

1) $v(a)=0, v(b)=0$.

2) $v(a)=0,\left.\frac{\partial F}{\partial u^{\prime}}\right|_{a} ^{b}-Q_{b}=0$.

3) $-\left.\frac{\partial F}{\partial u^{\prime}}\right|_{a}-Q_{a}=0, v(b)=0$.

4) $-\left.\frac{\partial F}{\partial u^{\prime}}\right|_{a}-Q_{a}=0,\left.\frac{\partial F}{\partial u^{\prime}}\right|_{b}-Q_{b}=0$.

Consider the problem of finding $(u, v)$, defined on a two dimensional region $\Omega$ such that the following functional is to be minimized:

$$
I(u, v)=\int_{\Omega} F\left(x, y, u, v, u_{x}, v_{x}, u_{y}, v_{y}\right) \mathrm{d} x \mathrm{~d} y
$$

with condition 


$$
\begin{gathered}
u=\hat{u} \text { so that } \delta u=0 \text { on } \Gamma \\
v=\hat{v} \text { so that } \delta v=0 \text { on } \Gamma \\
\frac{\partial F}{\partial u_{x}} \eta_{x}+\frac{\partial F}{\partial u_{y}} \eta_{y}=0 \text { on } \Gamma \\
\frac{\partial F}{\partial v_{x}} \eta_{x}+\frac{\partial F}{\partial v_{y}} \eta_{y}=0 \text { on } \Gamma .
\end{gathered}
$$

Equations (31) and (32) represent the essential boundary condition and Equations (33) and (34) represent the natural boundary conditions. The pair of elements $(u, v)$ are called the primary variables and

$$
Q_{x}=\frac{\partial F}{\partial x} \eta_{x}+\frac{\partial F}{\partial y} \eta_{y} \text { and } Q_{y}=\frac{\partial F}{\partial v_{x}} \eta_{x}+\frac{\partial F}{\partial v_{y}} \eta_{y}
$$

are secondary variables.

\subsubsection{Weak Form}

Weak form is defined to be a weighted integral statement of a differential equation in which the differentiation is transformed the dependent variable to the weight function such that all natural boundary conditions of the problem are also introduced.

\subsubsection{Ritz Method}

In Ritz method, the coefficients $c_{j}$ of the approximations are determined using the weak form of the problem, and hence choice of weight functions is restricted to the approximation function $w=\phi_{j}$.

Consider the variational problem resulting from the weak form: find the solution $u$ such that

$$
B(w, u)=l(w)
$$

for all sufficiently differentiable functions $w$ that satisfy the homogeneous form of the specified essential boundary conditions on $u$. In general, $B(;)$ can be unsymmetric in $w$ and $u$ and $l$ is linear, the problem in (36) is equivalent to minimization of the quadratic functional

$$
I(u)=\frac{1}{2} B(u, u)-l(u)
$$

In Ritz method, we seek an approximation solution to (36) in the form of a finite series

$$
U_{N}(x)=\sum_{j=1}^{N} c_{j} \phi_{j}(x)+\phi_{0}(x),
$$

where the constants $c_{j}$, called the Ritz coefficients are determined such that (36) holds for each

$$
w=\phi_{i}(i=1: N) \text {. }
$$

The function $\phi_{j}$ and $\phi_{0}$, called approximation functions are chosen such that $U_{N}$ satisfies the specified essential boundary conditions.

$$
B\left(\phi_{i}, \sum_{j=1}^{N} c_{j} \phi_{j}+\phi_{0}\right)=l\left(\phi_{i}\right), \quad(i=1: N) .
$$

Since $B(; \dot{)}$ is linear in $u$, we have

$$
\begin{gathered}
\sum_{j=1}^{N} B\left(\phi_{i}, \phi_{j}\right) c_{j}=l\left(\phi_{i}\right)-B\left(\phi_{i}, \phi_{j}\right) \\
\sum_{j=1}^{N} K_{i j} c_{j}=F_{i},(i=1: N) \\
K_{i j}=B\left(\phi_{i}, \phi_{j}\right), F=l\left(\phi_{i}\right)-B\left(\phi_{i}, \phi_{j}\right) .
\end{gathered}
$$

The algebraic equations in Equation (39) can be expressed in matrix form as:

$$
[K]\{c\}=[F] \text { or } K c=F \text {. }
$$

\subsubsection{Approximation Functions}

Let approximate solution is sought in the form

$$
U_{N}(x)=\sum_{j=1}^{N} c_{j} \phi_{j}(x)
$$

and suppose that the specified essential boundary conditions is $u\left(x_{0}\right)=u_{0}$, then $U_{N}$ must satisfy the condition $U_{N}\left(x_{0}\right)=u_{0}$ at a boundary point $x=x_{0}$ :

$$
\sum_{j=1}^{N} c_{j} \phi_{j}\left(x_{0}\right)=u_{0} \text {. }
$$

Since $c_{j}$ are unknown parameters to be determined, it is not easy to choose $\phi_{j}(x)$ such that the above relation holds. If $u_{0}$, then we cans select all $\phi_{j}$ such that $\phi_{j}\left(x_{0}\right)$ and satisfy the condition $U_{N}\left(x_{0}\right)=0$.

$$
\begin{gathered}
U_{N}\left(x_{0}\right)=\sum_{j=1}^{N} c_{j} \phi_{j}\left(x_{0}\right)+\phi_{0}\left(x_{0}\right) \\
u_{0}=\sum_{j=1}^{N} c_{j} \phi_{j}\left(x_{0}\right)+u_{0} \rightarrow \sum_{j=1}^{N} c_{j} \phi_{j}\left(x_{0}\right)=0
\end{gathered}
$$

which is satisfied, for arbitrary $c_{j}$, by choosing $\phi_{j}\left(x_{0}\right)=0$. If all specified essential boundary conditions are homogeneous, then $\phi_{0}$ is taken to be zero and $\phi_{j}$ must still satisfy the same conditions, $\phi_{j}\left(x_{0}\right)=0$, $j=1: N$. Note that the requirement that $w$ be zero at the boundary conditions are specified is satisfied by the choice $w=\phi_{j}(x)$.

\subsubsection{The Method of Weighted Residuals}

The method of weighted residual can be described in its generality by considering the operator equation

$$
A(u)=f \text { in } \Omega \text {. }
$$

where $A$ is an operator (linear or nonlinear), often a differential operator, acting on the dependent variables and $f$ is a known function of the independent variables.

In the weighted residual method, the solution $u$ is approximated, in much the same way as in Ritz method by the expression

$$
U_{N}\left(x_{0}\right)=\sum_{j=1}^{N} c_{j} \phi_{j}(x)+\phi_{0}(x)
$$


except that the requirements on $\phi_{0}$ and $\phi_{j}$ for the weighted residual method are more stringent than those for the Ritz method

$$
R=A\left(U_{N}\right)-f=A\left(\sum_{j=1}^{N} c_{j} \phi_{j}+\phi_{0}\right)-f \neq 0 .
$$

As the name suggests, the parameter $c_{j}$. are determined by requiring the residual $R$ to vanish in the weighted integral sense:

$$
\int_{\Omega} \psi_{i}(x) R\left(x, c_{j}\right) \mathrm{d} x \mathrm{~d} y .
$$

where $\psi_{i}(x)$ are weight functions. There are following type of well known method based on weight functions.

1) The Patrov-Galerkin method: The weighted residual method is referred as the Petrov-Galerkin method when $\psi_{i} \neq \phi_{i}$. When operator $A$ is linear Equation (47) is simplified to the form

$$
\begin{gathered}
\sum_{j=1}^{N}\left(\int_{\Omega} \psi_{i} A\left(\phi_{j}\right) \mathrm{d} x\right) c_{j}=\int_{\Omega} \psi_{i}\left[f-A\left(\phi_{0}\right)\right] \mathrm{d} x \\
\sum_{j=1}^{N} A_{i j} c_{j}=F_{i} \text { or } \quad(A C=F)
\end{gathered}
$$

where $A_{i j}=\int_{\Omega} \psi_{i} A\left(\phi_{j}\right) \mathrm{d} x$.

2) The Galerkin method: If the weight function $\psi_{i}$ is chosen to be equal to the approximation function $\phi_{i}$ the weighted residual method is better known as Galerkin method. The algebraic equations of the Galerkin approximation are

$$
\text { Ac }=F
$$

where

$$
A_{i j}=\int_{\Omega} \psi_{i} A\left(\phi_{j}\right) \mathrm{d} x, F_{i}=\int_{\Omega} \phi_{i}\left[f-A\left(\phi_{0}\right)\right] \mathrm{d} x,
$$

the approximation functions are used in Galerkin method required to be higher order than those in the Ritz method. For further readings we suggest to see [24-28].

3) The least-square method: In the least-square method, we determine the parameters $c_{j}$ by minimizing the integral of the square of the residual.

$$
\begin{gathered}
\frac{\partial}{\partial c_{i}} \int_{\Omega} R^{2}\left(x, c_{j}\right) \mathrm{d} x=0 \\
\int \frac{\partial R}{\partial c_{i}} R \mathrm{~d} x=0 .
\end{gathered}
$$

Here weight function $\psi_{i}=\frac{\partial R}{\partial c_{i}}$.

4) The collocation method: In the collocation method, we seek an approximate solution $U_{N}$ to Equation (44) in the form of $U_{N}$ by requiring the residual to vanish identically at $N$ selected points $x^{i}=\left(x^{i}, y^{i}, z^{i}\right)$ $(i=1: N)$ in the domain $\Omega$

$$
R\left(\boldsymbol{x}^{i}, c_{j}\right)=0 \quad(i=1: N) .
$$

The selection of points $\boldsymbol{x}^{i}$ is crucial in obtaining a well-conditioned system of equations and ultimately in obtaining an accurate solution. The collocation method can be shown to be a specified case of Equation (47) with $\psi_{i}=\delta\left(\boldsymbol{x}-\boldsymbol{x}^{i}\right)$, where $\delta(\boldsymbol{x})$ is the Dirac Delta fun- ctions which is defined by

$$
\int_{\Omega} f(x) \delta(x-\xi) \mathrm{d} x=f(\xi)
$$

with this choice of the weight functions, the weighted residual statement becomes

$$
\begin{gathered}
\int_{\Omega} \delta\left(\boldsymbol{x}-\boldsymbol{x}^{i}\right) R\left(\boldsymbol{x}, c_{j}\right) \mathrm{d} \boldsymbol{x}=0 \\
R\left(\boldsymbol{x}^{i}, c_{j}\right)=0 .
\end{gathered}
$$

The steps involved in finite element method of a problem:

1) Discretization of the given domain into a collection of prescribed finite element.

(a) Construct the finite element mesh of the prescribed elements.

(b) Number of nodes and elements.

(c) Generate the geometry properties needed for problem.

2) Derivation of element equations for all typical element in the mesh:

(a) Construct the variational formulation of the given differential equation over the typical element.

(b) Assume that a typical dependent variable $u$ is of the form

$$
u=\sum_{i=1}^{N} u_{i} \psi_{i}
$$

and substitute it into step 2(a) to obtain element equation in the form

$$
\left[K^{e}\right]\left[u^{e}\right]=\left[F^{e}\right] .
$$

(c) Select, if already available in the literature, or derive element interpolation functions $\psi_{i}$ and compute the element matrices.

3) Assembly of element equations to obtain the equation of whole problem.

4) Imposition of the boundary conditions of the problem.

5) Solution of the assembled equation.

6) Postprocessing of the results.

Authors can view the references [29-47] for more study on finite element method.

\section{Error Estimate}

In this section, we derive error estimates for the finite 
element method. The discrete version of the Generalized Lax-Milgram theorem, gives the uniqueness of the solution to the discrete equation and gives a first estimate of the error. This theorem is in fact only applicable when we use finite dimensional subspaces of our original Hilbert spaces. We have a more general case when we don't have such subspaces or when the operators in the variational equation are replaced by approximations (for instance by quadrature). We give also error estimates for this case. The error estimates depend on how good we can interpolate elements of Banach spaces subspaces of these Banach spaces, so we have to discuss the interpolation theory in Banach spaces, preceded by a necessary discussion of the formalism of the finite element method. This will give us estimates in the Sobolev norms $k: k m ; q ;$. We will also give an estimate in the $L^{2}$ norm, but for this we need additional requirements on the problem we consider.

1) The error estimate should give an accurate measure of the discretization error for a wide range of mesh spacings and polynomial degrees.

2) The procedure should be inexpensive relative to the cost of obtaining the finite element solution. This usually means that error estimates should be calculated using only local computations, which typically require an effort comparable to the cost of generating the stiffness matrix.

3) A technique that provides estimates of point-wise errors which can subsequently be used to calculate error measures in several norms is preferable to one that only works in a specific norm. Point-wise error estimates and error estimates in local (elemental) norms may also provide an indications as to where solution accuracy is insufficient and where refinement is needed.

A posteriori error estimates can roughly be divided into four categories:

1) Residual error estimates: Local finite element problems are created on either an element or a sub-domain and solved for the error estimate. The data depends on the residual of the finite element solution.

2) Flux-projection error estimates: A new flux is calculated by post processing the finite element solution. This flux is smoother than the original finite element flux and an error estimate is obtained from the difference of the two fluxes.

3) Extrapolation error estimates: Two finite element solutions having different orders or different meshes are compared and their differences used to provide an error estimate.

4) Interpolation error estimates: Interpolation error bounds are used with estimates of the unknown constants.

\section{Conclusions}

The present paper gives a comprehensive overview of the fundamentals of partial differential equations and related tools for their numerical solution available in the literature. Many fundamental ideas and techniques in finite difference and finite element methods have resemblance, and in some simple cases they coincide. Nevertheless, with its more systematic use of the variational approach, its greater flexibility, and the way it more easily lends itself to error analysis, the finite element method has become the dominating approach for tackling the partial differential equations together with their applications in science and engineering.

\section{Acknowledgements}

This research work is financially supported by the grant, No. SR/FTP/MS-14/2007, sponsored by Department of Science and Technology, New Delhi, Government of India. The authors would like to thank the anonymous reviewers for their valuable comments and suggestions to improve the manuscript.

\section{References}

[1] M. Kline, "Mathematical Thought from Ancient to Modern Times," Oxford University Press, London, 1972.

[2] H. Poincare, "Sur les Equations aux Derivees Partielles de la Physique Mathematique," American Journal of Mathematics, Vol. 12, No. 3, 1890, pp. 211-294. doi: $10.2307 / 2369620$

[3] H. Poincare, "Sur les Equations de la Physique MatheMatique," Rendiconti del Circolo Mathematico di Palermo, Vol. 8, 1894, pp. 57-155.

[4] E. E. Levi, "Sulle Equazioni Lineare Totalmente Ellitiche," Rendiconti del Circolo Mathematico di Palermo, Vol. 24, No. 1, 1907, pp. 275-317. doi:10.1007/BF03015067

[5] O. A. Ladyzhenskaya and N. N. Ural'tseva, "Linear and Quasi-Linear Elliptic Equations," Academic Press, New York, 1968.

[6] A. M. Micheletti and A. Pistoia, "On the Existence of Nodal Solutions for a Nonlinear Elliptic Problem on Symmetric Riemannian Manifolds," International Journal of Differential Equations, Vol. 2010, 2010, pp. 1-11. doi:10.1155/2010/432759

[7] I. M. Gelfand, "Some Problems in Theory of Quasilinear Equations," Transactions of the American Mathematical Society, Vol. 29, 1963, pp. 295-381.

[8] D. D. Joseph and E. M. Sparrow, "Nonlinear Diffusion Induced by Nonlinear Sources," Quarterly of Applied Mathematics, Vol. 28, 1970, pp. 327-342.

[9] H. B. Keller and D.S. cohen, "Some Positive Problems Suggested by Nonlinear Heat Generation," Journal of 
Mathematics and Mechanics, Vol. 16, No. 12, 1967, pp. 1361-1376.

[10] G. E. Forsthye and W. R. Wasow, "Finite Difference Methods for Partial Differential Equations," Wiley, New York, 1960.

[11] J. D. Hoffman, "Numerical Methods for Engineers and Scientists," 2nd Edition, McGraw-Hill, Inc., New York, 1992.

[12] M. K. Jain, R. K. Jain, "R. K. Mohanty, Fourth Order Difference Methods for the System of 2-D Non-Linear Elliptic Partial Differential Equations," Numerical Methods for Partial Differential Equations, Vol. 7, No. 3, 1991, pp. 227-244. doi:10.1002/num.1690070303

[13] L. V. Kantorovich and V. I. Krylov, "Approximate Methods in Higher Analysis," 3rd Edition, Interscience, New York, 1958.

[14] M. Kumar, P. Singh and P. Kumar, "A Survey on Various Computational Techniques for Nonlinear Elliptic Boundary Value Problems," Advances in Engineering Software, Vol. 39, No. 9, 2008, pp. 725-736. doi:10.1016/j.advengsoft.2007.11.001

[15] M. Kumar and P. Kumar, "Computational Method for Finding Various Solutions for a Quasilinear Elliptic Equation of Kirchhoff Type," Advances in Engineering Software, Vol. 40, No. 11, 2009, pp. 1104-1111. doi:10.1016/j.advengsoft.2009.06.003

[16] R. K. Mohanty and S. Dey, "A New Finite Difference Discretization of Order Four for $\partial u \partial n$ for Two-Dimensional Quasi-Linear Elliptic Boundary Value Problems," International Journal of Computer Mathematics, Vol. 76, No. 4, 2001, pp. 505-516. doi:10.1080/00207160108805043

[17] L. A. Ogenesjan and L. A. Ruchovec, "Study of the Rate of Convergence of Variational Difference Schemes for Second-Order Elliptic Equations in a Two-Dimensional Field with a Smooth Boundary," USSR Computational Mathematics and Mathematical Physics, Vol. 9, No. 5, 1969, pp. 158-183. doi:10.1016/0041-5553(69)90159-1

[18] R. D. Richtmyer and K. W. Morton, "Difference Methods for Initial Value Problems," 2nd Edition, Wiley-Interscience, New York, 1967.

[19] A. A. Samarskii, "Theory of Difference Schemes," Marcel Dekker Inc., New York, 2001.

[20] R. Eymard and T. R. Gallouët and R. Herbin, "The Finite Volume Method Handbook of Numerical Analysis," Vol. 7, 2000, pp. 713-1020. doi:10.1016/S1570-8659(00)07005-8

[21] R. J. Leveque, "Finite Volume Methods for Hyperbolic Problems," Cambridge University Press, Cambridge, 2002.

[22] P. Wesseling, "Principles of Computational Fluid Dynamics," Springer-Verlag, Berlin, 2001. doi:10.1007/978-3-642-05146-3

[23] I. Babuska, "Courant Element: Before and After," In: M. Krizek, P. Neittanmaki and R. Stenberg, Eds., Finite Element Methods: Fifty Years of the Courant Element, Marcel Dekker, New York, 1994, pp. 37-57.
[24] A. Pedasa and E. Tamme, "Discrete Galerkin Method for Fredholm Integro-Differential Equations with Weakly Singular Kernel," Journal of Computational and Applied Mathematics, Vol. 213, No. 1, 2008, pp. 111-126. doi:10.1016/j.cam.2006.12.024

[25] R. P. Kulkarni and N. Gnaneshwar, "Iterated Discrete Polynomially Based Galerkin Methods," Applied Mathematics and Computation, Vol. 146, No. 1, 2003, pp. 153165. doi:10.1016/S0096-3003(02)00533-7

[26] M. H. Schultz, "Rayleigh-Ritz-Galerkin Methods for MultiDimensional Problems," SIAM Journal on Numerical Analysis, Vol. 6, No. 4, 1969, pp. 523-538. doi: $10.1137 / 0706047$

[27] M. H. Schultz, " $L^{2}$ Error Bounds for the Rayleigh-RitzGalerkin Method," SIAM Journal on Numerical Analysis, Vol. 8, No. 4, 1971, pp. 737-748. doi:10.1137/0708067

[28] Y. Jianga and Y. Xu, "Fast Fourier Galerkin Methods for Solving Singular Boundary Integral Equations: Numerical Integration and Precondition," Journal of Computational and Applied Mathematics, Vol. 234, No. 9, 2010, pp. 2792-2807. doi:10.1016/j.cam.2010.01.022

[29] I. Babuska and A. K. Aziz, "Survey Lectures on the Mathematical Foundation of the Finite Element Method," In: A. K. Aziz, Ed., The Mathematical Foundations of the Finite Element Method with Applications to Partial Differential Equations, Academic Press, New York, 1972, pp. 5-359.

[30] K. Böhmer, "Numerical Methods for Nonlinear Elliptic Differential Equations," Oxford University Press, New York, 2010.

[31] R. Courant, "Variational Methods for the Solution of Problems of Equilibrium and Vibration," Bulletin of American, Mathematical Society, Vol. 49, 1943, pp. 1-23. doi:10.1090/S0002-9904-1943-07818-4

[32] M. Ghimenti and A. M. Micheletti, "On the Number of Nodal Solutions for a Nonlinear Elliptic Problems on Symmetric Riemannian Manifolds," Electronic Journal of Differential Equations, Vol. 18, 2010, pp. 15-22.

[33] N. Hirano, "Multiple Existence of Solutions for a Nonlinear Elliptic Problem on a Riemannian Manifold," Nonlinear Analysis: Theory, Methods and Applications, Vol. 70, No. 2, 2009, pp. 671-692.

[34] D. V. Hutton, "Fundamentals of Finite Element Analysis," Tata McGraw-Hill, New York, 2005.

[35] M. Kumar and P. Kumar, "A Finite Element Approach for Finding Positive Solutions of Semilinear Elliptic Dirichlet Problems," Numerical Methods for Partial Differential Equations, Vol. 25, No. 5, 2009, pp. 1119-1128. doi:10.1002/num.20390

[36] M. Kumar and P. Kumar, "Simulation of a Nonlinear Steklov Eigen Value Problem Using Finite Element Approximation," Computational Mathematics and Modelling, Vol. 21, No.1, 2010, pp. 109-116. doi:10.1007/s10598-010-9058-6

[37] R. Molle, "Semilinear Elliptic Problems in Unbounded Domains with Unbounded Boundary," Asymptotic Analysis, Vol. 38, No. 3-4, 2004, pp. 293-307. 
[38] J. T. Oden and D. Somogyi, "Finite Element Applications in Fluid Dynamics," Journal of the Engineering Mechanics Division, ASCE, Vol. 95, No. 4, 1968, pp. 821-826.

[39] J. T. Oden, "A General Theory of Finite Elements, II: Applications," International Journal for Numerical Methods in Engineering, Vol. 1, No. 3, 1969, pp. 247-259. doi:10.1002/nme.1620010304

[40] J. T. Oden, "A Finite Element Analogue of the NavierStokes Equations," Journal of the Engineering Mechanics Division, ASCE, Vol. 96, No. 4, 1970, pp. 529-534.

[41] E. R. De Arantes and E. Oliveira, "Theoretical Foundation of the Finite Element Method," International Journal of Solids and Structures Vol. 4, No. 10, 1968, pp. 926952.

[42] S. S. Rao, "The Finite Element Method in Engineering," 4th Edition, Elsevier, Butterworth Heinemann, 2005.
[43] J. N. Reddy, "An Introduction to the Finite Element Method," 3rd Edition, McGraw-Hill, New York, 2005.

[44] M. Ramos and H. Tavares, "Solutions with Multiple Spike Patterns for an Elliptic System," Calculus of Variations and Partial Differential Equations, Vol. 31, No. 1, 2008, pp. 1-25. doi:10.1007/s00526-007-0103-Z

[45] F. Williamson, "A Historical Note on the Finite Element Method," International Journal for Numerical Methods in Engineering, Vol. 15, No. 6, 1980, pp. 930-934. doi:10.1002/nme.1620150611

[46] O. C. Zienkiewicz, "The Finite Element Method in Engineering Science," 3rd Edition, Mc-Graw-Hill, London, 1977.

[47] M. Zlamal, "On the Finite Element Method," Numerische Mathematik, Vol. 12, No. 5, 1968, pp. 394-409. doi:10.1007/BF02161362 